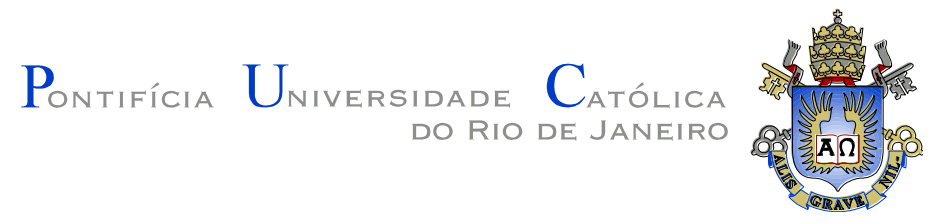

Marcelo de Mello Camanho

\title{
A Model for Stream-based Interactive Storytelling
}

Thesis presented to the Programa de PósGraduação em Informática, of the Departamento de Informática do Centro Técnico Científico da PUCRio, as partial fulfillment of the requirements for the degree of Doutor.

Advisor: Prof. Bruno Feijó

Rio de Janeiro

April, 2014 
Marcelo de Mello Camanho

\title{
A Model for Stream-based Interactive Storytelling
}

Thesis presented to the Programa de Pós-Graduação em Informática, of the Departamento de Informática do Centro Técnico Científico da PUC-Rio, as partial fulfillment of the requirements for the degree of Doutor.

\author{
Prof. Bruno Feijó \\ Advisor \\ Departamento de Informática - PUC-Rio
}

Antonio Luz Furtado

Departamento de Informática - PUC-Rio

Angelo Ernani Maia Ciarlini

UNIRIO

Luis Paulo Santos Valente

PUC-Rio

Sean Wolfgand Matsui Siqueira

UNIRIO

Prof. José Eugenio Leal

Coordinator of the Centro Técnico Científico da PUC-Rio 
All rights reserved.

\section{Marcelo de Mello Camanho}

Graduated in Information Systems in UNIRIO (Universidade Federal do Estado do Rio de Janeiro) in 2005 and obtained a degree of Mestre em Informática at UNIRIO in 2009. He has participated in multiple congresses on Digital Entertainment and studies the interactive storytelling area since graduation.

Bibliographic Data

Marcelo de Mello Camanho

A Model for Stream-based Interactive Storytelling / Marcelo de Mello Camanho ; orientador: Bruno Feijó. - 2014.

92 f. : il. ; $30 \mathrm{~cm}$

Tese (doutorado)-Pontifícia Universidade Católica do Rio de Janeiro, Departamento de Informática, 2014. Inclui bibliografia

1. Informática - Teses. 2. Inteligência Artificial. 3. Votação. 4. Interactive Storytelling. 5. Massive Digital Entertainment. 6. Streaming. 7. Cross-media games. 8. TV. I. Feijó, Bruno II. Pontifícia Universidade Católica do Rio de Janeiro. Departamento de Informática. III. Título.

CDD: 004 


\section{Acknowledgments}

To my family, girlfriend and friends for their support, care and love, essential to have come this far.

To my advisor for helping the research and believing it was possible to finish this work.

I am also thankful to the members of this work's examining committee, who have contributed generously with their time and expertise.

To CAPES and PUC-Rio, for the granted support, a help that made this work possible. 


\section{Abstract}

Camanho, Marcelo; Feijó, Bruno. A Model for Stream-based Interactive Storytelling. Rio de Janeiro, 2014. 92p. DSc Thesis - Departamento de Informática, Pontifícia Universidade Católica do Rio de Janeiro.

In this thesis we present a highly scalable architecture for massive multiuser interactive storytelling systems based on video streams. The proposed architecture can support different demands for interactivity, generation, and visualization of stories in digital television environments, which include TV settop boxes, tablets, smartphones, and computers. In this architecture, the same story adapts itself to the spectator's device in terms of rendering and interface processes automatically. Also a model for sharing massive interactive stories is presented. Moreover, the proposed system preserves the logical coherence of the story that unfolds while keeping it interactive.

\section{Keywords}

Interactive Storytelling, Massive Digital Entertainment, Streaming, Crossmedia games, TV. 


\section{Resumo}

Camanho, Marcelo; Feijó, Bruno. Um modelo para storytelling interativo baseado em streaming de video. Rio de Janeiro, 2014. 92p. DSc Thesis Departamento de Informática, Pontifícia Universidade Católica do Rio de Janeiro.

Nesta tese é apresentada uma arquitetura altamente escalável para storytelling interativo em massa baseado em streams de vídeo. A arquitetura proposta pode suportar diferentes demandas para interatividade, geração e visualização de histórias em ambientes de televisão digital, que inclui set-top boxes de TV, tablets, smartphones e computadores. Nesta arquitetura, a mesma história se adapta ao aparelho do espectador em termos de renderização e processo de interface automaticamente. Também é apresentado um modelo para compartilhar histórias interativas em massa. Além disso, o sistema proposto preserva a coerência lógica da história que se desenrola enquanto a mantém interativa.

\section{Palavras-chave}

Storytelling Interativo, Entretetenimento Digital em Massa, Streaming, Jogos multiplataforma, TV. 


\section{Contents}

1 Introduction 11

2 Theoretical Background 15

$\begin{array}{ll}\text { 2.1. Interactive Storytelling } & 15\end{array}$

2.2. Logtell 20

2.2.1. Story Generation $\quad 21$

2.2.2. Step-by-step mode $\quad 27$

2.2.3. Continuous Flow 28

2.2.4. Dramatization 30

2.3. Digital Television 31

2.3.1. Interactivity and Television 34

2.3.2. Applications and Services in Interactive Television 35

2.4. Voting Systems 38

2.4.1. Basic definitions 39

2.4.2. Criteria and Methods 41

2.4.3. Weighted voting systems 43

2.4.4. Some final remarks on voting systems $\quad 45$

3 A Model for Stream Based Interactive Storytelling 47

3.1. Multiplatform Interactive Storytelling Model 47

3.2. A Model for Sharing Massive Interactive Stories 52

3.3. Voting Strategies $\quad 54$

3.3.1. User Model and Voter Importance $\quad 55$

3.3.2. Voting Strategies and Methods $\quad 57$

3.4. Voting Harmonization 63

3.5. Streaming Capabilities $\quad 65$

4 Processes and Methods $\quad 66$

4.1. Application environment 66 
4.2. Drama Streamer lifecycle $\quad 67$

$\begin{array}{ll}\text { 4.3. Prototype Clients } & 69\end{array}$

4.4. New Server Interface 73

4.5. Evaluation and Tests 75

4.5.1. Performance and architecture 75

4.5.2. Voting Strategies Tests $\quad 77$

4.6. Conclusions 81

5 Conclusions and Future Work 83

5.1. Main Contributions 83

5.2. Future Work 86

6 References $\quad 89$ 


\section{Lista de figuras}

$\begin{array}{lr}\text { Figure } 1 \text { Story Cicle } & 12\end{array}$

Figure 2 Network environment of digital television for massive multiplayer $\begin{array}{ll}\text { interactive storytelling systems } & 13\end{array}$

Figure 3 Sleep is Death [10] 16

$\begin{array}{ll}\text { Figure } 4 \text { Façade [11] } & 17\end{array}$

Figure 5 - Twitch plays Pokemon [34] 19

Figure 6 Logtell Current Architecture $\quad 21$

Figure 7 Logtell Step-by-step Mode 26

Figure 8 Logtell's continuous interaction 29

Figure 9 Logtell 3D dramatization $\quad 30$

Figure 10 Simplified stream-based interactive storytelling architecture $\quad 48$

Figure 11 The proposed architecture for the stream-based interactive storytelling system 49

Figure 12 Simplified Class Diagram With new overall model 51

Figure 13 Scheduling a streamed story 53

Figure 14 Simple Suggestion / User Model 55

Figure 15 New continuous flow and rewards 56

Figure 16 Voting Strategies $\quad 57$

Figure 17 Highest Score Voting Strategy 58

Figure 18 Most Voted Voting Strategy $\quad 59$

Figure 19 Simple User Chosen Vote Strategy 59

Figure 20 Simple User Good Vote Strategy $\quad 60$

Figure 21 Weighted Chosen Good Vote Strategy 61

$\begin{array}{ll}\text { Figure 22 Probability Voting } & 61\end{array}$

Figure 23 Balanced Square Root $\quad 62$

Figure 24 Voting Harmonization 63

Figure 25 Sorted Voting Harmonization $\quad 64$

Figure 26 Drama Streamers $\quad 65$

Figure 27 Activity diagram for the process of watching a story 67 
Figure 28 different story client menus (Android and Windows)

$\begin{array}{ll}\text { Figure } 29 \text { Multiple story clients } & 70\end{array}$

Figure 30 Logtell Twitch Streaming $\quad 71$

Figure 31 Twitch client using Logtell 72

Figure 32 Streaming Server $\quad 84$

Figure 33 Multiple Clients $\quad 85$ 